\title{
A Comparison of Error Rates Between Intravenous Push Methods: A Prospective, Multisite, Observational Study
}

\author{
John B. Hertig, PharmD, MS, CPPS, * Daniel D. Degnan, PharmD, MS, CPPS, CPHQ, * \\ Catherine R. Scott, CPHQ, * Janelle R. Lenz, PharmD, * \\ Xiaochun Li, PhD, MSc, $\dagger$ and Chelsea M. Anderson, PharmD, MBA, BCPS*
}

\begin{abstract}
Objectives: Current literature estimates the error rate associated with the preparation and administration of all intravenous (IV) medications to be $9.4 \%$ to $97.7 \%$ worldwide. This study aims to compare the number of observed medication preparation and administration errors between the only commercially available ready-to-administer product (Simplist) and IV push traditional practice, including a cartridge-based syringe system (Carpuject) and vials and syringes.

Methods: A prospective, multisite, observational study was conducted in 3 health systems in various states within the United States between December 2015 and March 2016 to observe IV push medication preparation and administration. Researchers observed a ready-to-administer product and IV push traditional practice using a validated observational method and a modified data collection sheet. All observations were reconciled to the original medication order to determine if any errors occurred.

Results: Researchers collected 329 observations (ready to administer $=102$; traditional practice $=227$ ) and observed 260 errors (ready to administer $=25$; traditional practice $=235$ ). The overall observed error rate for ready-to-administer products was $2.5 \%$, and the observed error rate for IV push traditional practice was $10.4 \%$.

Conclusions: The ready-to-administer group demonstrated a statistically significant lower observed error rate, suggesting that use of this product is associated with fewer observed preparation and administration errors in the clinical setting. Future studies should be completed to determine the potential for patient harm associated with these errors and improve clinical practice because it relates to the safe administration of IV push medications.
\end{abstract}

Key Words: IV push medication administration, direct observation, medication errors, safety, error rates, ready-to-administer IV push medication, nurse administration

(J Patient Saf 2018;14: 60-65)

The he use of intravenous (IV) medications is essential to providing patient care in the hospital setting. An estimated $44 \%$ of nurses administer injectable medications more than 5 times per shift. ${ }^{1}$ However, these products carry a high potential for patient harm because of the number of complex manipulations required to administer, compatibility concerns, narrow therapeutic indexes,

From the *Center for Medication Safety Advancement, Purdue University College of Pharmacy, Fishers; and †Department of Biostatistics, School of Medicine \& Richard M. Fairbanks School of Public Health, Indiana University, Indianapolis, IN

Correspondence: Chelsea M. Anderson, PharmD, MBA, BCPS, Center for

Medication Safety Advancement, Purdue University College of Pharmacy, Fishers, IN (e-mail: canderson@purdue.edu)

This study was funded by BD Rx Inc (later acquired by Fresenius Kabi). The funder played no role in the design and conduct of the study; collection, management, analysis, and interpretation of the data; or preparation, review, or approval of this manuscript.

The authors disclose no conflict of interest.

Copyright (C) 2017 The Author(s). Published by Wolters Kluwer Health, Inc. This is an open-access article distributed under the terms of the Creative Commons Attribution-Non Commercial-No Derivatives License 4.0 (CCBY-NC-ND), where it is permissible to download and share the work provided it is properly cited. The work cannot be changed in any way or used commercially without permission from the journal. and challenges with reversing pharmacologic effects of drugs administered by this route. ${ }^{2}$ This risk is recognized by the medical community, and $99 \%$ of nurses agree that errors related to IV medication use pose a serious risk to patients. ${ }^{1}$ In addition, many IV medications were identified as having a serious risk for patient harm on the high-alert medication list for acute care settings. ${ }^{3}$

A study in 2012 by Lahue et al $^{4}$ estimated that 1.2 million hospitalizations each year are impacted by preventable adverse drug events associated with injectable medications. Almost half (48\%) of the errors that occur with all IV medications happen during preparation or administration, ${ }^{1}$ but error rates related to these practices vary significantly in the literature. Studies worldwide estimate the error rate with all IV medications to be between $9.4 \%$ and $97.7 \%$, with IV push administrations demonstrating higher error rates than IV infusions. ${ }^{5-12}$ Common IV medication errors included failure to maintain aseptic technique during drug preparation, use of the wrong diluent, and incorrect labeling of an IV product. $^{5-12}$

One of the factors associated with an increased potential for error with IV medications is the number of complex manipulations required when preparing and administering these drugs. ${ }^{2,13}$ Drug manufacturers have begun to develop and market ready-toadminister IV push products with the aim of reducing this complexity of drug preparation and administration, while minimizing the potential for errors and patient harm. Ready-to-administer products are viewed as the IV drug delivery systems of choice, particularly because of their low risk for contamination and ease of use. ${ }^{14,15}$ They are recommended for use in procedural areas and for dispensing anesthetic products to help reduce errors while maintaining efficiency. ${ }^{15-18}$ Although there is support for the use of these products, the authors could not identify any literature quantifying error rates between the available IV push methods in actual practice. One study using a simulated method did find a reduction in medication errors when using ready-to-administer products; however, the authors of the simulation study note potential limitations when applied to the clinical setting, suggesting that further research needs to be conducted to validate their findings. ${ }^{19}$

The primary objective of this study was to compare the number of observed medication preparation and administration errors between the only commercially available ready-to-administer product (Simplist; Fresenius Kabi, Lake Zurich, Ill) ${ }^{20}$ and IV push traditional practice, including a cartridge-based syringe system (Carpuject; Hospira, Lake Forest, Ill) ${ }^{21}$ and vials and syringes. Secondary objectives included further analysis of the observed error rate and identifying specific types of errors observed within each group of the study. The researchers hypothesized that use of a ready-to-administer product would result in fewer observed medication errors because of the reduced complexity of manipulations required to administer the product as intended.

\section{METHODS}

A prospective, multisite, observational study of IV push medication preparation and administration was conducted in 3 health 
systems in various states within the United States between December 2015 and March 2016. This study was approved by both the Purdue University Institutional Review Board (IRB) and through each study site's own specific review process. Informed consent was obtained from all nursing personnel before participation in the study.

The following inclusion criteria were used to select study site candidates: use of the only commercially available ready-toadminister product (Simplist), accreditation by The Joint Commission, Magnet designation from the American Nurses Credentialing Center, and a Hospital Safety Score (generated by The Leapfrog Group) of $\mathrm{C}$ or greater. These inclusion criteria were used as objective measures to identify sites with robust safety cultures. Sites were excluded if they were pediatric units or had previously used the AU MEDS (MedAccuracy, LLC, Auburn University, Auburn, Ala) system. The AU MEDS system is a validated observational method designed to collect data through direct observation at the point of medication preparation and administration. $^{22}$ Excluding sites with previous AU MEDS use helped ensure consistency in staff response to observers, AU MEDS processes, and relative observation methods. Each site was contacted by a study team member and completed a letter of interest articulating their desire to participate in the study. After review, all sites accepted the Purdue University IRB approval and 1 site obtained additional approval from a site-specific IRB.

This observational research included 2 study groups to assess differences in observed errors and error rates associated with ready-to-administer products compared with traditional practice. The first group consisted of observations of medication preparation and administration of a ready-to-administer product (Simplist). The second group consisted of observations of medication preparation and administration of IV push traditional practice,

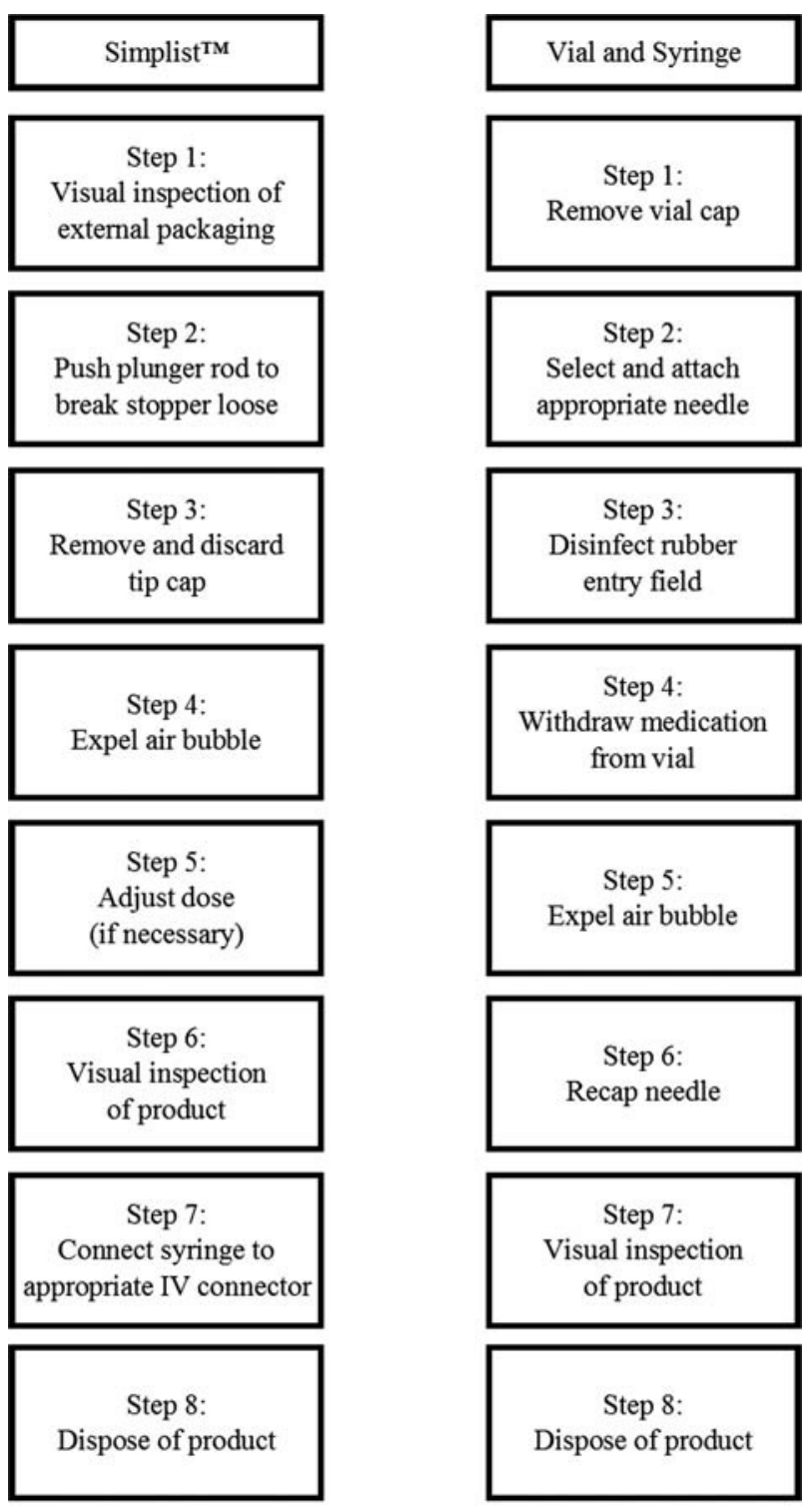

* Opportunities for error also included inappropriate dilution of product and labeling error

FIGURE 1. Opportunities for error by IV push method. 
TABLE 1. Interrater Agreement as Measured by Cohen $\mathrm{k}$

\begin{tabular}{lc}
\hline Rater Pairs & Cohen $\kappa$ \\
\hline Observer A and B & 0.93 \\
Observer A and C & 0.93 \\
Observer B and C & 0.87 \\
\hline
\end{tabular}

including a cartridge-based syringe system (Carpuject) and vial and syringe. It was estimated that 240 observations of both readyto-administer and IV push traditional practice (with approximately 120 per technique) would sufficiently power the study ( $\beta$ at 0.8 ) to detect a difference of $15 \%$ in observed errors in medication preparation and administration between the 2 study groups.

Observations were collected using AU MEDS, and all researchers successfully completed the AU MEDS training program and exam. Researchers who participated in the data collection also completed shared observations at a separate site not included in the study, and the percentage agreement on each observation was used to calculate a Cohen $\kappa$ interrater reliability score.

Researchers directly observed medication preparation and administration at 3 separate study sites. Nurses were observed after removal of the IV push medication from the automated dispensing cabinets (ADCs) to administration of the dose to the patient. Observation typically occurred between $8 \mathrm{AM}$ and $6 \mathrm{PM}$ to capture as many doses as possible, based on ADC dispensing data. Only patient care areas that were serviced by ADCs were included in this study.

Before on-site observations, researchers defined the steps required in a correct IV push medication preparation and administration process for each IV push method by completing 3 distinct failure modes and effects analyses (FMEAs) for each observed method. An FMEA, or a step-wise approach used to identify all of the possible ways a process may fail, was developed for the Simplist, vial and syringe, and Carpuject process by breaking down all steps perceived necessary for successful administration of medication using information from manufacturer package inserts and best practice guidelines. These steps were objectively discussed and debated by the research team, and each FMEA was adapted to create an observational research form, which was used to collect relevant data at each study site. Both study groups included IV push methods that had 10 steps (Fig. 1) or opportunities for error based on this impartial, objective analysis (the Carpuject method required 13 steps, however all but 1 Carpuject vial used without the Carpuject cartridge. Therefore, those observations were coded as a 10-step vial and syringe administration).

Data regarding completion of each of the technique steps along with the following information were collected: date of preparation and administration, unit, hospital, observer, patient code, time when medication was removed from the ADC, administration time, medication given, and comments. All observations were reconciled to the original medication order and the predefined process steps to determine if an error occurred.

\section{Definition of Error and Error Rate}

For this study, an error was defined as a deviation of the observed medication preparation and administration from the previously defined steps or any deviation from the original medication order. The overall observed error rate was defined as the total number of technique errors divided by the total number of opportunities for error

\section{Definition of Dilution and Dilution Error}

Dilution was defined as any IV push medication that was diluted with normal saline or other diluent before administration to the patient. Dilution did not include reconstituting powdered medication with the appropriate diluent (e.g., pantoprazole). Dilution errors were defined as dilution occurring when no dilution was indicated. Dilution errors also included no dilution occurring when dilution was indicated in site-specific policy or the medication administration record.

\section{Statistics}

The average number of errors per administration was calculated by study group, both overall and within each study site. The chance of committing any errors per observation was compared between the 2 study groups using the Fisher exact test. Statistically significant differences were defined as $P<0.05$. The number of errors per observation (with a total number of opportunities for errors being 10 for each group or 13 for the 1 observed Carpuject administration) was compared between the 2 techniques using a stratified log-linear model, using the 3 sites as strata. Cohen $\kappa$ as an index of interrater agreement between 2 raters on categorical data was calculated for each pair of the 3 raters. The desired interrater reliability was defined as a Cohen $\kappa$ coefficient of greater than 0.8 .

\section{RESULTS}

To determine the observed error rate for IV push medication preparation and administration of the ready-to-administer products and IV push traditional practice, researchers directly observed this process at 3 separate sites for a total of 337 hours. Researchers collected a total of 329 observations (ready to administer $=102$; traditional practice $=227$ ) and observed a total of 260 errors (ready to administer $=25$; traditional practice $=235$ ). The Cohen $\kappa$ calculated for interrater agreement was greater than or equal to 0.867 for each researcher pair (Table 1).

Ready-to-administer products were associated with fewer observed errors than IV push traditional practice. Only 22 of 102 ready-to-administer $(22 \%)$ observations contained errors versus 179 of 227 traditional practice $(79 \%)$ observations contained errors $(P<0.001)$.

Observed error rates were calculated using total number of errors divided by total number of opportunities for error. To control for potential site differences, a stratified log-linear analysis with site as strata was performed. This analysis confirmed a uniformly significant lower observed error rate for ready-to-administer products at each site $(P<0.02)$, allowing the data to be pooled for an aggregate analysis. Overall, site B demonstrated a lower observed error rate for both ready-to-administer and traditional practice than site $\mathrm{A}$ or $\mathrm{C}$; however, the error rate for ready to

TABLE 2. Average of Number of Errors per Opportunity by Site and Group

\begin{tabular}{llcc}
\hline Site & \multicolumn{1}{c}{ Group } & Error Rate & $\boldsymbol{P}$ \\
\hline All sites & Ready to administer & 0.025 & $<0.02$ \\
All sites & Traditional practice & 0.104 & \\
Site A & Ready to administer & 0.020 & 0.01 \\
Site A & Traditional practice & 0.126 & \\
Site B & Ready to administer & 0.004 & 0.02 \\
Site B & Traditional practice & 0.044 & \\
Site C & Ready to administer & 0.034 & $<0.001$ \\
Site C & Traditional practice & 0.105 &
\end{tabular}

$P$ values are for group comparisons within site, based on a stratified model with site as strata. 


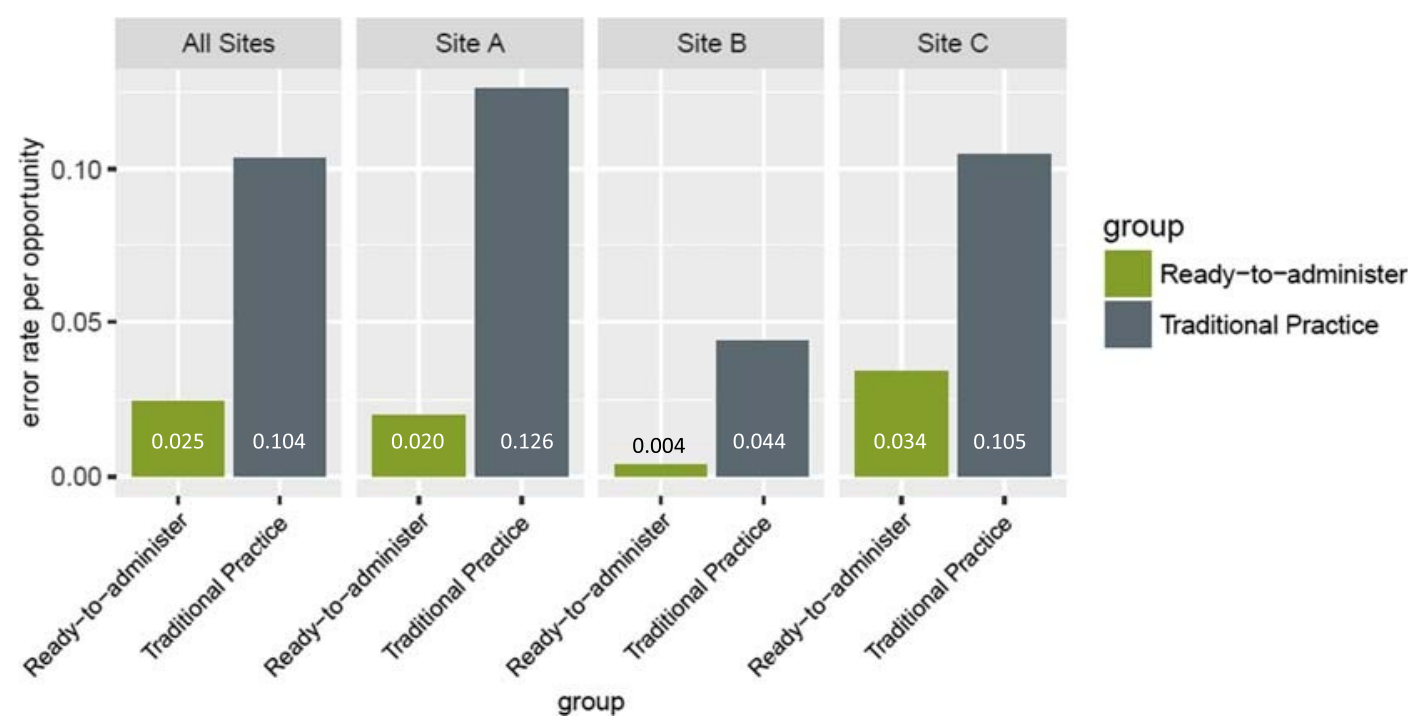

FIGURE 2. Error rate per opportunity, overall and by site.

administer was still significantly lower than traditional practice $(P=0.018)$ (Table 2$)$. The overall observed error rate for ready to administer was $2.5 \%$ (25 errors and 1020 opportunities for error), and the observed error rate for IV push traditional practice was $10.4 \%$ (235 errors and 2270 opportunities for error) (Fig. 2).

There were a total of 21 labeling errors (ready to administer $=3$; traditional practice $=18$ ), 85 dilution errors (ready to administer $=21$; traditional practice $=64$ ), and 153 disinfection errors (ready to administer $=0$; traditional practice $=153$ ). . Medications diluted more than $50 \%$ of the time during the observations included diphenhydramine, famotidine, hydromorphone, hydralazine, ketorolac, labetalol, lorazepam, metoclopramide, and promethazine. Specific dilution observed error totals are noted in Table 3.

A total of 65 IV push medication administrations with a Carpuject vial were observed during the study. The Carpuject vial was used with a Carpuject cartridge and administered as directed by the package insert during 1 observation and was used and recorded as a vial and syringe administration during the other 64 observations.

\section{DISCUSSION}

Ready-to-administer products were developed with the goal of reducing the complexity of IV push medication administration and improving safe medication use practices of injectable products. ${ }^{23}$ The primary objective of this research was to compare the number of observed medication preparation and administration errors between the only commercially available ready-toadminister product and IV push traditional practice. The results demonstrate that there is a statistically significant difference between ready-to-administer and IV push traditional practice, with fewer errors associated with preparation and administration of ready-to-administer products.

\section{Risk Points and Primary Objective}

Counting the number of steps in the ready-to-administer process and comparing that absolute number against the number of steps required to administer a medication via vial and syringe may not accurately represent the risk of a given process. For example, a step in the Simplist process requires the user to "push plunger rod to break stopper loose." This step arguably carries less risk of harm to the patient when compared with select steps in the vial and syringe process including "select and attach appropriate needle" or "disinfect rubber entry field." In reviewing each process step for the study groups, the relative risk points and likelihood of patient harm should be assessed against total number of steps. ${ }^{15}$ In addition, the study results suggest that these points of higher risk found in the traditional practice group of the study were also associated with higher rates of errors. The lower observed error rate at site B may be attributed to the smaller overall number of observations collected there compared with sites $\mathrm{A}$ and $\mathrm{C}$.

\section{Secondary Objectives}

Secondary objectives for this research included further analysis of the specific types of errors observed within each study group including extra dose, omission, unordered medication, wrong

TABLE 3. Total Number of Dilutions and Dilution Errors by Drug

\begin{tabular}{lccc}
\hline Drug & $\begin{array}{c}\text { Dilution Error } \\
\text { (\% of Total) }\end{array}$ & $\begin{array}{c}\text { Dilution } \\
\text { (\% of Total) }\end{array}$ & $\begin{array}{c}\text { Total } \\
\text { Observations }\end{array}$ \\
\hline Ondansetron & $19(32)$ & $25(42)$ & 59 \\
Hydromorphone & $18(21)$ & $65(76)$ & 86 \\
Diphenhydramine & $16(50)$ & $18(56)$ & 32 \\
Ketorolac & $9(50)$ & $9(50)$ & 18 \\
Famotidine & $6(86)$ & $6(86)$ & 7 \\
Promethazine & $6(32)$ & $17(89)$ & 19 \\
Morphine & $4(7)$ & $29(48)$ & 61 \\
Metoclopramide & $2(50)$ & $2(50)$ & 4 \\
Lorazepam & $1(33)$ & $2(67)$ & 3 \\
Methylprednisolone & $1(13)$ & $2(25)$ & 8 \\
Hydralazine & $1(100)$ & $1(100)$ & 1 \\
Labetalol & $1(100)$ & $1(100)$ & 1 \\
Furosemide & $1(10)$ & $4(40)$ & 10 \\
Pantoprazole & 0 & $3(27)$ & 11 \\
Chlorothiazide & 0 & $0(0)$ & 2 \\
Metoprolol & 0 & $0(0)$ & 2 \\
Thiamine & 0 & $0(0)$ & 2 \\
Dexamethasone & 0 & $0(0)$ & 1 \\
Prednisolone & 0 & $0(0)$ & 1 \\
\hline
\end{tabular}


dose, wrong form, wrong route, wrong time, and wrong technique. Errors associated with disinfection, dilution, and labeling were included as a wrong technique error.

There were 3 labeling errors with ready-to-administer products compared with 18 errors with IV push traditional practice. These results suggest that using vial and syringe practice requires additional manipulation, which can result in leaving an unlabeled syringe unattended. Previous studies suggest that unlabeled medications can be given by mistake because of confusion about syringe contents and potentially result in patient harm. ${ }^{2,15}$ The findings in the current study regarding the lack of syringe labeling are similar to findings in a study that observed nursing practice in a simulated environment. ${ }^{19}$ The ready-to-administer products in this study are labeled by the manufacturer eliminating the need for this step before medication administration. The 3 errors observed as part of this study occurred because nursing staff withdrew the medication from the Simplist syringe and then injected the medication into an unlabeled flush syringe, which was left unlabeled and unattended. The Joint Commission's National Patient Safety Goals outline best practices with labeling medications, indicating that all medications, medication containers, and other solutions on and off the sterile field in perioperative and other procedural settings should be labeled. ${ }^{24}$ There have been cases of serious harm reported when patients were accidentally administered flush syringes that included medication but were left unattended and unlabeled. ${ }^{16}$ The Institute for Safe Medication Practices released a "Medication Safety Alert" outlining the risks associated with unlabeled IV medications and also provided a best practices document to help organizations minimize risks associated with improperly labeled products. ${ }^{16}$

Disinfecting and sterilizing all potential points of contamination are key factors in preventing nosocomial infections. Prevention of these types of infections is a priority for hospitals and healthcare systems, and various state and federal organizations have included infection-related metrics into quality programs. ${ }^{25}$ A risk point identified in this study was the disinfection of the vial stopper before puncture and withdrawal of the medication in the traditional practice research group. Best practice suggests the rubber stopper of medication vials is disinfected with sterile $70 \%$ alcohol before the insertion of a needle to protect against the transfer of microorganisms from the healthcare provider or environment to the patient. ${ }^{25}$ The study results demonstrated 106 errors resulting from failure to disinfect the vial stopper in the traditional practice group, whereas no errors were observed in the ready-to-administer group because this product required no additional disinfecting before administration to the patient. This difference is clinically significant, given the safety risks associated with contaminated medications

The unnecessary dilution of medications, particularly ready-toadminister products, continues to be a topic of debate among those involved in best practice development. In many care settings, there seems to be a practice disposition toward dilution, rather than determining a specific need for dilution on a drugby-drug or case-by-case basis. ${ }^{26}$ The United States Pharmacopeia Chapter $\langle 797>$, a standard for compounded sterile preparations, indicates that any transfer of medication from 1 device to another presents a potential contamination and safety risk. ${ }^{27}$ Health professionals should seek to minimize these transfers, attempting to complete the successful administration of a medication in the fewest steps necessary. Researchers observed a total of 47 readyto-administer and 137 traditional practice dilutions throughout the duration of this study. Per study definitions, 21 ready-toadminister dilutions and 64 traditional practice dilutions were deemed to be errors. Although both study groups demonstrated a clinically significant number of dilutions, the ready-to-administer product was associated with fewer of these types of errors. There are a variety of safety issues associated with dilution including an increased likelihood of unlabeled and mislabeled syringes, the possibility of contamination, the potential for dosing errors, and an increased likelihood for precipitate formation with the use of an incompatible diluent. ${ }^{2,15}$ Best practices advise that IV push medications should only be diluted when recommended by the manufacturer, supported by evidence in peer-reviewed biomedical literature, or in accordance with institutional guidelines. ${ }^{15}$ The package inserts for Simplist products do not instruct healthcare professionals to dilute before administration, with the exception of doses that exceed what is commercially available in the prepackaged syringes. ${ }^{20}$ Specifically, the manufacturer recommends not to "introduce any other fluid into the syringe at any time." Study researchers found differences in site-specific guidance documents when completing preparatory research because institutional policies are not uniform in nature. Researchers also found dilution suggestions within such places as the medication administration record or built within the physician order sets. Coordination between nursing, pharmacy, and medicine departments is encouraged to ensure that dilution only occurs when necessary for optimal patient care.

\section{Other Errors Observed}

The inclusion of the cartridge-based syringe system (Carpuject) into this study resulted in 65 observed uses of Carpuject across the 3 sites. There was only a single observation of a Carpuject being used as intended with the Carpuject cartridge equating to a $1.5 \%$ success rate with this process. In 64 cases, the Carpuject was misused by neglecting to attach the necessary cartridge, resulting in the device being used as a vial. To ensure accurate study documentation, as soon as a Carpuject was used as a vial, the study observer assessed the remaining administration procedure using the vial process observation tool. This ensured a consistent approach to error detection by assuming that a Carpuject used as a vial could still serve utility. Despite the small number of cartridge-based syringe system observations, the low observed success rate would seem to indicate that there is a need for additional education and training on these devices if they are to be used in a manner consistent with manufacturer guidelines.

\section{Remaining Gaps and Further Research}

This research study was designed to assess the use of ready-toadminister products when compared with traditional practice, with a specific focus on technique errors. The results from these primary and secondary measures have provided insights not previously reported in the literature. The information learned has subsequently highlighted additional gaps in available knowledge that were outside the scope of this study.

Further research is needed to assess the true impact of harm resulting from the errors identified in this study. An outcomes study linking the risks of each process step with resulting patient harm from the errors would help further demonstrate the impact on patient safety. Additional gaps in IV push administration center around the decision to dilute medications, and there is a need for research and education regarding appropriate dilution practices. Further research should identify key stakeholders, including frontline healthcare professionals, medication safety experts, and clinical leaders to determine appropriate guidelines for effective and safe dilution. In addition, further exploration into the relative safety of ready-to-administer products as related to infection-control considerations is warranted. This study demonstrated a clinically significant difference in appropriate disinfection when the readyto-administer products group was compared with traditional practice. 
Determining the risk and patient impact of inappropriate disinfection, accompanied by evidenced-based recommendations, would assist hospitals and health systems in their journey toward achieving a comprehensive culture of cleanliness.

\section{Limitations}

Statistical analysis confirmed that the 3 sites were similar enough to pool for data analysis; however, no study site is exactly the same and there may be potential differences in the culture, processes, and people who were involved in administering IV push medications. Moreover, variability in individual site policies and procedures surrounding dilution practices determined whether a dilution was categorized as an error or an appropriate dilution. Changes in these policies or procedures would impact the final error calculations. Observations were also collected sequentially as opposed to randomly, which may have affected the observed error rate. Finally, the number of steps chosen to be observed in each group was developed from an FMEA analysis and any change in the number of these process steps has the potential to alter the final calculated error rates.

\section{CONCLUSIONS}

Intravenous push medication preparation and administration of ready-to-administer products and traditional practice, including a cartridge-based syringe system and vial and syringes, were observed in a prospective, multisite, observational study. The ready-to-administer group had an observed error rate of $2.5 \%$, whereas the traditional practice group had an observed error rate of $10.4 \%$. This difference was found to be statistically significant and would suggest that use of ready-to-administer products is associated with fewer observed errors in the study's clinical settings. This study addressed key questions while identifying additional gaps in the available literature surrounding IV push medication practice. Future studies should be completed to determine the potential for patient harm associated with these errors and improve clinical practice because it relates to the safe administration of IV push medications.

\section{REFERENCES}

1. American Nurses Association. Study of Injectable Medication Errors. 2007. http://www.nursingworld.org/FunctionalMenuCategories/ MediaResources/PressReleases/2007/SyringeSafetyStudy.pdf. Accessed April 28, 2016

2. Grissinger M. Reducing errors with injectable medications: unlabeled syringes are surprisingly common. $P$ T. 2010;35:428-451.

3. ISMP. ISMP List of High-Alert Medications in Acute-Care Settings. 2014. Available at: https://www.ismp.org/Tools/ highalertmedications.pdf. Accessed April 28, 2016.

4. Lahue BJ, Pyenson B, Iwasaki K, et al. National burden of preventable adverse drug events associated with inpatient injectable medications: healthcare and medical professional liability costs. Am Health Drug Benefits. 2012;5:1-10.

5. Fahimi F, Ariapanah P, Faizi M, et al. Errors in preparation and administration of intravenous medications in the intensive care unit of a teaching hospital: an observational study. Aust Crit Care. 2008;21: $110-116$.

6. Abbasinazari M, Hajhossein Talasaz A, Mousavi Z, et al. Evaluating the frequency of errors in preparation and administration of intravenous medications in orthopedic, general surgery, and gastroenterology wards of a teaching hospital in Tehran. Iran J Pharm Res. 2013;12:229-234.
7. Bruce J, Wong I. Parenteral drug administration errors by nursing staff on an acute medical admission ward during day duty. Drug Saf. 2001;24: $855-862$.

8. Ding Q, Barker KN, Flynn EA, et al. Incidence of intravenous medication errors in a Chinese hospital. Value Health Reg Issues. 2015;33-39.

9. Taxis K, Barber N. Ethnographic study of incidence and severity of intravenous drug errors. BMJ. 2003;326:684.

10. Westbrook JI, Rob MI, Woods A, et al. Errors in the administration of intravenous medications in hospital and the role of correct procedures and nurse experience. BMJ Qual Saf. 2011;20:1027-1034.

11. Ong WM, Subasyini S. Medication errors in intravenous drug preparation and administration. Med J Malaysia. 2013;69:52-57.

12. al Tehewy M, Fahim H, Gad NI, et al. Medication administration errors in a university hospital. J Patient Saf. 2016;12:34-39.

13. Reporting and Learning Services, National Patient Safety Agency, National Health Service. Promoting Safer Use of Injectable Medicines-Risk Assessment Tool. 2007. Available at: www.nrls.npsa.nhs.uk/resources/? entryid45=59812. Accessed April 28, 2016.

14. Sanborn MD, Moody ML, Harder KA, et al. Second consensus development conference on the safety of intravenous drug delivery systems -2008. Am J Health Syst Pharm. 2009;66:185-192.

15. ISMP. ISMP Safe Practice Guidelines for Adult IV Push Medications. 2015. Available at: http://www.ismp.org/Tools/guidelines/ivsummitpush/ ivpushmedguidelines.pdf. Accessed April 28, 2016.

16. ASHP Advantage E-newsletter. Outsourcing Sterile Compounding Services and Prefilled Anesthesia Syringes. 2010. Available at: http://www.ashpadvantage.com/outsource/outsource-winter-nl.pdf. Accessed April 28, 2016.

17. Fraind DB, Slagle JM, Tubbesing VA, et al. Reengineering intravenous drug and fluid administration processes in the operating room: step one: task analysis of existing processes. Anesthesiology. 2002;97:139-147.

18. Australian and New Zealand College of Anaesthetists. Guidelines for the Safe Administration of Injectable Drugs in Anaesthesia. 2009. Available at: http://www.anzca.edu.au/documents/ps51-2009-guidelines-for-the-safeadministration-o.pdf. Accessed April 28, 2016.

19. Burger M, Degnan D. Comparative safety, efficiency, and nursing preference among 3 methods for intravenous push medication preparation: a randomized crossover simulation study. J Patient Saf. 2016. [Epub ahead of print]

20. BD Rx Inc. BD Simplist ${ }^{\mathrm{TM}}$ package insert. 2012

21. Hospira. Carpuject Sterile Cartridge Unit package insert. 2006.

22. Flynn EA, Barker KN, Pepper GA, et al. Comparison of methods for detecting medication errors in 36 hospitals and skilled-nursing facilities. Am J Health Syst Pharm. 2002;59:436-446.

23. Fresenius Kabi. BD Simplist ${ }^{\mathrm{TM}}$ Prefilled Syringes. BD Rx Web site. Available at: http://www.bdrxinc.com/bd-simplist.asp. Accessed April 22, 2016.

24. The Joint Commission. Standards BoosterPak ${ }^{\mathrm{TM}}$ for Safe Medication Storage, MM.03.01.01. Updated April 2014. Available at: http://www. wrnmmc.capmed.mil/Shared $\% 20$ Documents/Survey $\% 20$ Readiness $\%$ 20Resources/Medication\%20Storage\%20Booster\%20Pak.pdf. Accessed April 28, 2016.

25. Centers for Disease Control and Prevention. Injection Safety. Last updated March 2, 2011. Available at: http://www.cdc.gov/injectionsafety/providers/ provider_faqs_med-prep.html. Accessed April 22, 2016.

26. Heindel GA, Stivers AP. Culture changes needed to implement ISMP IV push guidelines. Hosp Pharm. 2017;52:167-168.

27. U.S. Pharmacopeia. United States Pharmacopeia and National Formulary (USP 33-NF 28 Reissue) [CD-ROM]. Rockville, MD: US Pharmacopeia; 2010; chap 797. 